\title{
UTE-T2* Analysis of Diseased and Healthy Achilles Tendons and Correlation with Clinical Score: An In Vivo Preliminary Study
}

\author{
Yang Qiao, ${ }^{1}$ Hong-Yue Tao, ${ }^{1}$ Kui Ma, ${ }^{2}$ Zi-Ying $\mathrm{Wu},{ }^{2}$ Jian-Xun $\mathrm{Qu},{ }^{3}$ and Shuang Chen ${ }^{1}$ \\ ${ }^{1}$ Department of Radiology, Huashan Hospital, Fudan University, Shanghai, China \\ ${ }^{2}$ Department of Sports Medicine, Huashan Hospital, Shanghai, China \\ ${ }^{3}$ GE Healthcare Applied Science Lab, Shanghai, China
}

Correspondence should be addressed to Shuang Chen; chenshuang6898@126.com

Received 26 July 2016; Accepted 24 November 2016; Published 5 January 2017

Academic Editor: Haining Zhang

Copyright (C) 2017 Yang Qiao et al. This is an open access article distributed under the Creative Commons Attribution License, which permits unrestricted use, distribution, and reproduction in any medium, provided the original work is properly cited.

\begin{abstract}
Objective. To compare $2^{*}$ value of healthy and diseased Achilles tendons (AT) with a recently introduced three-dimensional ultrashort echo time (3D-UTE) sequence and analyze the correlation between T2* value and clinical scores. Methods. Ten patients with symptomatic Achilles tendon and ten healthy volunteers were investigated with 3D-UTE sequence on a 3T magnetic resonance (MR) scanner. T2* values of four regions in Achilles tendons were calculated. The clinical outcomes of patients were evaluated according to the American Orthopaedic Foot and Ankle Society (AOFAS) score and Achilles Tendon Rupture Score (ATRS). An independent sample $t$-test was used to compare the differences of $\mathrm{T} 2{ }^{*}$ value and clinical scores between two groups. The Pearson correlation coefficient between clinical scores and T2* values was assessed. Results. The T2* values of Achilles tendon were statistically significantly different between patients and volunteers. The Pearson correlation coefficients between T2* and AOFAS or ATRS scores of patients were $r=-0.733$ and $r=-0.634$, respectively. Conclusion. The variability of T2* in healthy and pathologic AT can be quantified by UTE-T2* ${ }^{*} 2^{*}$ may be a promising marker to detect and diagnose AT tendinopathy. UTE-T2* could give a precise guidance to clinical outcome.
\end{abstract}

\section{Introduction}

Tendinopathy, a syndrome with tendon pain, tenderness, and swelling that limited the tendon function, is one of the most common injures in both athletic and nonathletic populations $[1,2]$. Nowadays, due to a higher involvement in sports, the prevalence of Achilles tendinopathy is increasing [3]. Although the exact etiology is still uncertain, it is supposed that repetitive overload and overuse are the major causes [4]. They may lead to irreversible degenerative changes of the Achilles tendon (AT), for instance, destruction and decrease of collagen fiber in extracellular matrix, increased vascularity, and altered cellularity [5-7]. Consequently, a precise way to detect Achilles tendinopathy is highly demanded.

As a noninvasive and credible diagnostic tool, magnetic resonance imaging (MRI) has been widely used to evaluate the pathological changes of AT. But highly organized AT with very short T2 appear dark on conventional MRI sequences, for only tissues with long T2 relaxation times could be visualized $[8,9]$. Thus a short echo time sequence is needed to acquire signal from the AT. Three-dimensional ultrashort echo time (UTE) imaging, with an echo time as short as 0.05$0.5 \mathrm{~ms}$, provided direct visualization and quantitative $\mathrm{T} 2{ }^{*}$ mapping for short-T2* components [10-12]. Biochemical changes of early stage Achilles tendinopathy may affect $\mathrm{T} 2{ }^{*}$ values of AT and thus can be caught and quantified with UTE sequences [8].

Therefore, the aim of this study was to investigate the capability of quantitative 3D-UTE-T2* in evaluating diseased $\mathrm{AT}$ and analyze the correlation between $\mathrm{T} 2{ }^{*}$ value and American Orthopaedic Foot and Ankle Society (AOFAS) score or Achilles tendon Total Rupture Score (ATRS). We hypothesize that the pathologic AT would show increased $\mathrm{T}^{*}$ value while comparing with matched healthy samples and $\mathrm{T}^{*}$ value may be correlated with clinical score. 


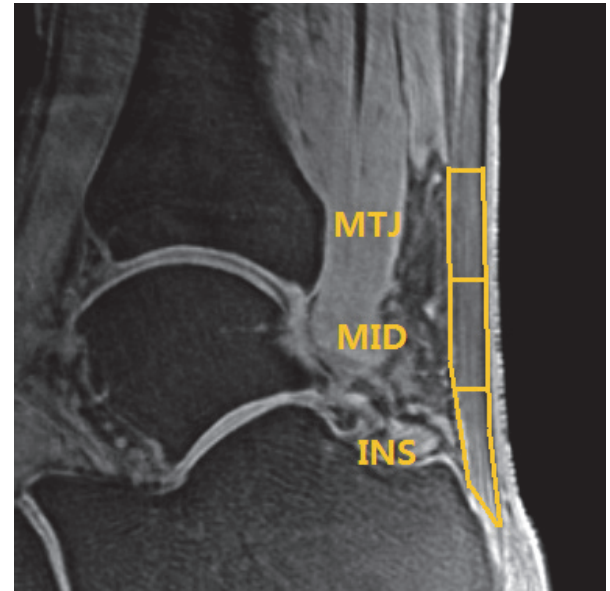

FIGURE 1: ROIs of Achilles tendon on sagittal UTE image acquired at $\mathrm{TE}=0.032 \mathrm{~ms}$. INS $=$ insertion, $\mathrm{MID}=$ middle, $\mathrm{MTJ}=$ muscletendon junction; these three ROIs were equally divided according to the longitudinal length of Achilles tendon. The bulk ROI consisted of all the three ROIs.

\section{Materials and Methods}

2.1. Participants. The study was approved by the institutional review board of our hospital and all participants' informed consents were obtained. Ten patients $(9$ male $/ 1$ female, mean age $37.10 \pm 8.60$ years, BMI $\left.23.00 \pm 2.15 \mathrm{~kg} / \mathrm{m}^{2}\right)$ with pain or abnormalities in the AT and ten healthy volunteers matched for sex, age, and BMI ( 9 male/ 1 female, mean age $37.40 \pm 10.61$ years, BMI $23.94 \pm 2.32 \mathrm{~kg} / \mathrm{m}^{2}$ ) participated in the study. Participants were excluded if they had significant tendon rupture or any contraindication for MR.

2.2. MRI and Clinical Evaluation. All the participants were examined on a 3T MR scanner (Discovery 750, GE Healthcare, Waukesha, WI, USA) to get monoexponential calculation of $\mathrm{T}^{*}$ in the human AT in vivo. As a quantitative $3 \mathrm{D}$ UTE sequence, four echo times ( $\mathrm{TE}=0.032,7.5,20.5$, and $28 \mathrm{~ms}$ ) were acquired. The parameters were set as follows: sagittal orientation, FOV $=140 \times 140 \mathrm{~mm}$, slice thickness = $2.0 \mathrm{~mm}$, flip angle $=18$, and number of excitations (NEX) $=1$. Fat-saturated proton-density weighted turbo-spin echo (PD-TSE) sequence was underwent to acquire morphological assessment with the parameters: sagittal orientation, TR $2843.0 \mathrm{~ms}$, FOV $180 \times 180 \mathrm{~mm}$, slice thickness $2.0 \mathrm{~mm}$, flip angle 142, and number of excitations $(\mathrm{NEX})=2$.

For clinical evaluation, AOFAS scoring system and ATRS were used to evaluate the patients' clinical outcome (0-100 points, worst to best).

2.3. Imaging Analysis. Images from the UTE-T2* sequence were analyzed by software in the work station of GE. The AT was segmented and divided into three parts equally according to length: insertion (INS), middle (MID), and muscle-tendon junction (MTJ) (Figure 1). These three ROIs as well as all bulk of AT regions on each echo of UTE-T2* images were drawn to get the mean MR signal. T2* value of each region is calculated by fitting the acquired signal at different echo time to a single exponential decay model (Figure 2).

2.4. Statistical Analysis. All statistical analyses were performed in SPSS 20.0 (SPSS Institute, Chicago, IL, USA). An independent sample $t$-test was used to compare the differences of T2* values between two groups. Pearson's correlation coefficient was used to analyze correlations between clinical scores and $\mathrm{T}^{*}$ values of patients. The difference would be statistically significant if $P$ value $<0.05$.

\section{Results}

There were no obvious tendon tears on MRI for all patients. The mean T2* value for bulk ROIs was significantly higher in patients than that in volunteers $(12.508 \pm 0.940$ and $11.081 \pm$ 0.297, $P=0.001$ ) (Table 1). Separately, MTJ, MID, and INS regions of patients had statistically higher T2* value compared with the matched regions of volunteers (MTJ: $11.977 \pm 0.831$ and $11.005 \pm 0.581, P=0.007$, MID: $12.474 \pm$ 1.261 and $11.124 \pm 0.394, P=0.008$, and INS: $13.124 \pm$ 0.943 and $11.084 \pm 0.522, P=0.000)$. The difference in INS region is greater than that in MID and MTJ. In patients, the mean AOFAS and ATRS were $70.6 \pm 5.58$ and $52.8 \pm 8.27$, respectively. The $\mathrm{T}^{*}$ value for bulk region was negatively correlated with AOFAS as well as ATRS score $(r=-0.733$, $P=0.016$, and $r=-0.634, P=0.049$ ) (Figure 3).

In this study, $\mathrm{T}^{*}$ relaxation time in pathologic and healthy AT was measured using UTE-T2* sequence and a significant higher $\mathrm{T} 2{ }^{*}$ value was observed in all four regions of diseased AT. Besides, T2* value of all bulk of AT in patients was found to be negatively correlated with AOFAS and ATRS score.

\section{Discussion}

UTE-T2* mapping, a novel quantitative technique, could catch the short-T2* relaxations from AT that are not well captured by standard T2 mapping [13]. In the early stages of Achilles tendinopathy, it is usually biochemical but not morphological changes that are found [14], which consist of destruction of collagen structure and increase of proteoglycan and water content [15]. UTE-T2* mapping is sensitive to these changes; thus it could be a useful tool to detect tendon disease in an early stage [12]. The results of this study suggest that the variability of Achilles tendinopathy can be quantified by UTE-T2* . The increasing of $\mathrm{T} 2{ }^{*}$ value may due to disorganization of collagen structure and increasing of water content in tendons. What is more, the difference in INS region is greater. The reason could be that the enthesis is mostly involved in overuse injuries of AT $[16,17]$. Gardin et al. [18] applied monoexponential calculation and showed a significant higher $\mathrm{T} 2{ }^{*}$ relaxation time in symptomatic tendons compared with control tendons. In a study by Juras et al., they compared mono- and biexponential T2* analysis using variable-echo time sequence (vTE) and found that increased $\mathrm{T}^{*}$ in all parts of diseased AT with monoexponential analysis [11]. Juras et al. also reported a similar finding with bicomponent quantitative 3D-UTE. They found 


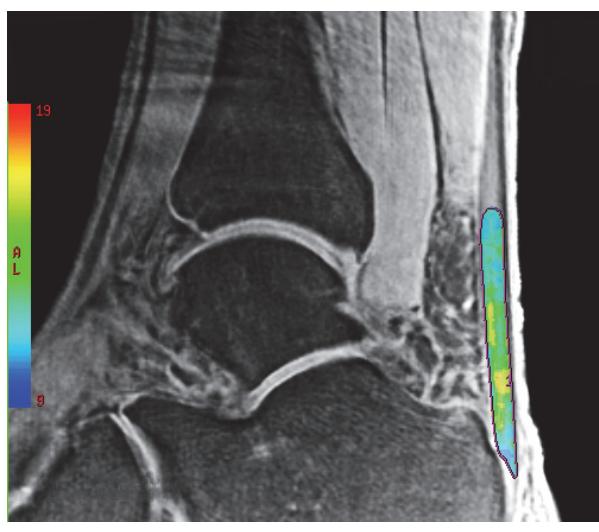

(a)

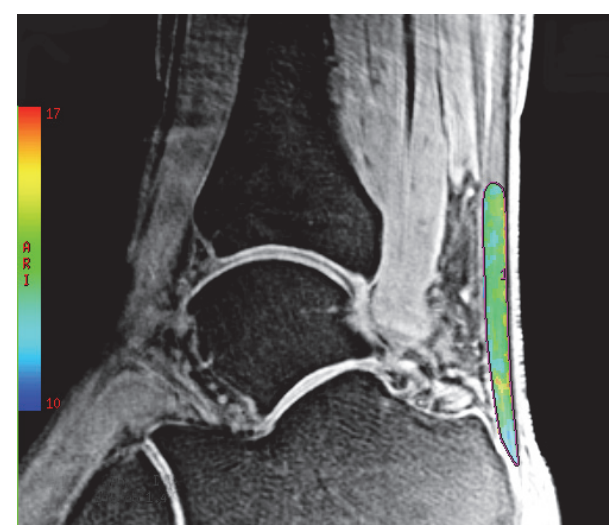

(b)

Figure 2: UTE T2* mapping on diseased (a) and healthy (b) Achilles tendons of a 36-year-old male acquired at TE $=0.032 \mathrm{~ms}$. Color scale represents $\mathrm{T} 2{ }^{*}$ values. The increase of $\mathrm{T} 2{ }^{*}$ values was observed in diseased tendon.

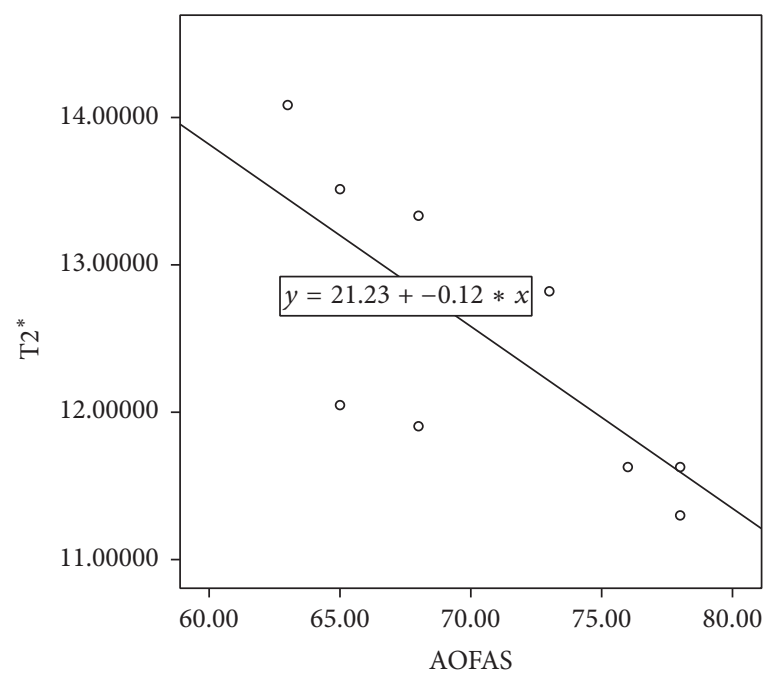

(a)

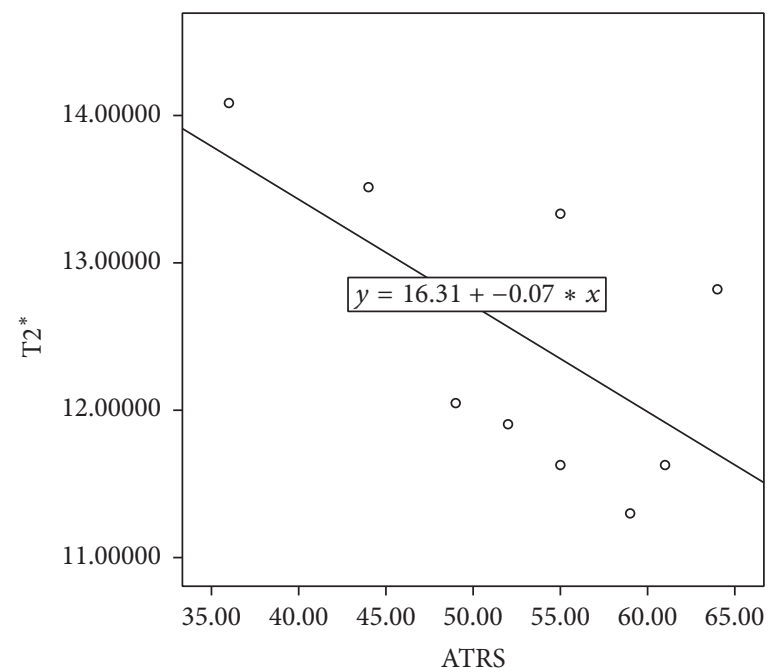

(b)

Figure 3: (a) A scatter plot of the bulk T2* and AOFAS of ten patients. The Pearson correlation coefficient was $r=-0.733(P=0.016)$. (b) A scatter plot of the bulk T2* and ATRS of ten patients. The Pearson correlation coefficient was $r=-0.634(P=0.049)$.

significant differences between healthy $(10.28 \pm 2.28 \mathrm{~ms})$ and degenerated AT $(12.85 \pm 1.87 \mathrm{~ms})$ in the long component of $\mathrm{T} 2{ }^{*}$ [7]. While estimating the diagnostic value of $\mathrm{T} 1$ and $\mathrm{T} 2{ }^{*}$ relaxation times and off-resonance saturation ratio, Grosse et al. also observed statistical significant differences between the patients with tendinopathy and controls [19].

We applied monoexponential calculation of UTE-T2* ${ }^{*}$. Juras et al. [11] found that the short component of $\mathrm{T} 2{ }^{*}$ reflects the changes of Achilles tendinopathy more accurately than the monoexponential T2* [11]. However, owing to the longer scanning time and higher sensitivity to movements and the magic angle, biexponential calculation is more difficult to be applied clinically [18].

To the best of our knowledge, only a few studies analyzed the correlation between $\mathrm{T}_{2}{ }^{*}$ value and clinical score. Both AOFAS and ATRS scores are widely used in clinical practice and validated in many studies $[20,21]$. They had general assessment of the AT situation. T2* value of the bulk region in patients was correlated with AOFAS and ATRS score, which suggests that $\mathrm{T}^{*}$ could give a precise guidance to clinical outcome of patients with Achilles tendinopathy. Juras et al. got a similar correlation between ATRS score and the monoexponential $\mathrm{T}^{*}$ [11].

There are also some limitations in the study. Firstly, a small number of patients which would lead to increased statistical deviation. The patient cohort would be enlarged in our next study. Secondly, monoexponential calculation of $\mathrm{T} 2{ }^{*}$ reflects the mean value of all the components of relaxation time, which may lead to an underrate of $\mathrm{T} 2^{*}$, especially in diseased tendons [7].

\section{Conclusion}

In conclusion, the differences between $\mathrm{T}_{2}{ }^{*}$ in healthy and pathologic tendons could be observed by UTE-T2* ${ }^{*}$. As the preliminary patient data suggest, $\mathrm{UTE}-\mathrm{T} 2{ }^{*}$ is an acute marker 
TABLE 1: Comparison of T2* values between patients and volunteers. Mean (M) and standard deviation (SD) as well as $P$ value were presented. The $P$ value applied the significant difference in the four parts. Mean $(\mathrm{M})$ and standard deviation (SD) of AOFAS and ATRS scores were also presented.

\begin{tabular}{lcccccc}
\hline & & \multicolumn{2}{c}{ Patients } & & Volunteers & SD \\
& & M & SD & M & 11.081 & 0.297 \\
T2* values & Bulk & 12.508 & 0.940 & 11.005 & 0.581 & 0.001 \\
& MTJ & 11.977 & 0.831 & 11.124 & 0.394 & 0.007 \\
AOFAS score & MID & 12.474 & 1.261 & 11.084 & 0.008 \\
ATRS score & INS & 13.124 & 0.943 & & \\
\hline
\end{tabular}

to detect AT tendinopathy in the early stage and it gives a precise guidance to clinical outcome. By further investigation in larger cohort of patients, different terms of follow-up after treatments are required to define the exact role of UTE-T2* for monitoring the change of AT.

\section{Competing Interests}

The authors declare that there is no conflict of interests regarding the publication of this paper.

\section{Authors' Contributions}

Yang Qiao and Hong-Yue Tao contributed equally to this work and should be considered co-first authors.

\section{Acknowledgments}

This project was subsidized by the National Science Foundation for Distinguished Young Scholars of China (no. 81501440) and the Project of Shanghai Municipal Science and Technology Commission (16ZR1404600).

\section{References}

[1] S. G. Rees, C. M. Dent, and B. Caterson, "Metabolism of proteoglycans in tendon," Scandinavian Journal of Medicine and Science in Sports, vol. 19, no. 4, pp. 470-478, 2009.

[2] A. Möller, M. Åström, and N. E. Westlin, "Increasing incidence of Achilles tendon rupture," Acta Orthopaedica Scandinavica, vol. 67, no. 5, pp. 479-481, 1996.

[3] S. L. James, B. T. Bates, and L. R. Osternig, "Injuries to runners," The American Journal of Sports Medicine, vol. 6, no. 2, pp. 40-50, 1978.

[4] P. Kannus and L. Jozsa, "Histopathological changes preceding spontaneous rupture of a tendon: a controlled study of 891 patients," Journal of Bone and Joint Surgery A, vol. 73, no. 10, pp. 1507-1525, 1991.

[5] M. Aström and A. Rausing, "Chronic Achilles tendinopathy. A survey of surgical and histopathologic findings," Clinical Orthopaedics and Related Research, no. 316, pp. 151-164, 1995.

[6] T. Movin, A. Gad, F. P. Reinholt, and C. Rolf, "Tendon pathology in long-standing achillodynia. Biopsy findings in 40 patients," Acta Orthopaedica Scandinavica, vol. 68, no. 2, pp. 170-175, 1997.

[7] V. Juras, S. Zbyn, C. Pressl et al., "Regional variations of $\mathrm{T}_{2}^{*}$ in healthy and pathologic achilles tendon in vivo at 7 Tesla: preliminary results," Magnetic Resonance in Medicine, vol. 68, no. 5, pp. 1607-1613, 2012.

[8] S. Peto, P. Gillis, and V. P. Henri, "Structure and dynamics of water in tendon from NMR relaxation measurements," Biophysical Journal, vol. 57, no. 1, pp. 71-84, 1990.

[9] M. D. Robson, P. D. Gatehouse, M. Bydder, and G. M. Bydder, "Magnetic resonance: an introduction to ultrashort TE (UTE) imaging," Journal of Computer Assisted Tomography, vol. 27, no. 6, pp. 825-846, 2003.

[10] P. D. Gatehouse and G. M. Bydder, "Magnetic resonance imaging of short T2 components in tissue," Clinical Radiology, vol. 58, no. 1, pp. 1-19, 2003.

[11] V. Juras, S. Apprich, P. Szomolanyi, O. Bieri, X. Deligianni, and S. Trattnig, "Bi-exponential T2* analysis of healthy and diseased Achilles tendons: an in vivo preliminary magnetic resonance study and correlation with clinical score," European Radiology, vol. 23, no. 10, pp. 2814-2822, 2013.

[12] M. D. Robson, M. Benjamin, P. Gishen, and G. M. Bydder, "Magnetic resonance imaging of the Achilles tendon using ultrashort TE (UTE) pulse sequences," Clinical Radiology, vol. 59, no. 8, pp. 727-735, 2004.

[13] E. Y. Chang, J. Du, and C. B. Chung, "UTE imaging in the musculoskeletal system," Journal of Magnetic Resonance Imaging, vol. 41, no. 4, pp. 870-883, 2015.

[14] S. Tom, J. Parkinson, M. Z. Ilic, J. Cook, J. A. Feller, and C. J. Handley, "Changes in the composition of the extracellular matrix in patellar tendinopathy," Matrix Biology, vol. 28, no. 4, pp. 230-236, 2009.

[15] G. H. Filho, J. Du, B. C. Pak et al., "Quantitative characterization of the Achilles tendon in cadaveric specimens: T1 and T2* measurements using ultrashort-TE MRI at 3 T," American Journal of Roentgenology, vol. 192, no. 3, pp. W117-W124, 2009.

[16] M. Kvist, "Achilles tendon injuries in athletes," Sports Medicine, vol. 18, no. 3, pp. 173-201, 1994.

[17] M. Benjamin, H. Toumi, J. R. Ralphs, G. Bydder, T. M. Best, and S. Milz, "Where tendons and ligaments meet bone: attachment sites ('entheses') in relation to exercise and/or mechanical load," Journal of Anatomy, vol. 208, no. 4, pp. 471-490, 2006.

[18] A. Gardin, P. Rasinski, and J. Berglund, "T2* relaxation time in achilles tendinosis and controls and its correlation with clinical score," Journal of Magnetic Resonance Imaging, vol. 43, pp. 14171422, 2016.

[19] U. Grosse, R. Syha, T. Hein et al., "Diagnostic value of T1 and $\mathrm{T} 2{ }^{*}$ relaxation times and off-resonance saturation effects in the evaluation of achilles tendinopathy by MRI at 3T," Journal of Magnetic Resonance Imaging, vol. 41, no. 4, pp. 964-973, 2015. 
[20] L. J. Lapidus, B. A. Ray, and P. Hamberg, "Medial Achilles tendon island flap-a novel technique to treat reruptures and neglected ruptures of the Achilles tendon," International Orthopaedics, vol. 36, no. 8, pp. 1629-1634, 2012.

[21] R. S. Kearney, J. Achten, S. E. Lamb, C. Plant, and M. L. Costa, "A systematic review of patient-reported outcome measures used to assess Achilles tendon rupture management: what's being used and should we be using it?" British Journal of Sports Medicine, vol. 46, no. 16, pp. 1102-1109, 2012. 


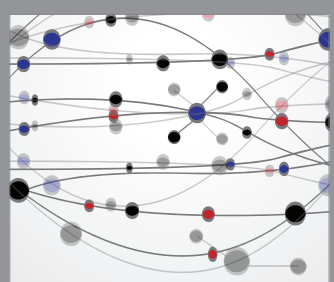

The Scientific World Journal
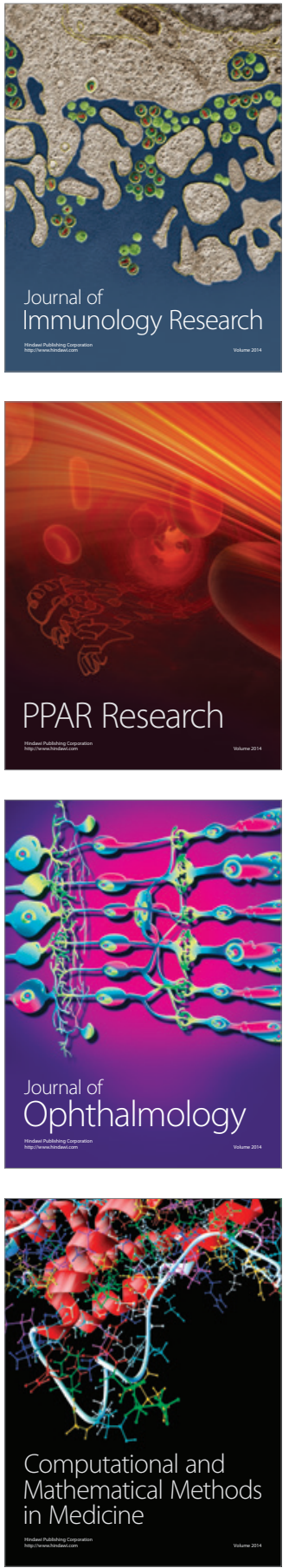

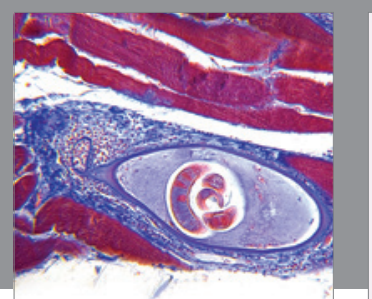

Gastroenterology Research and Practice
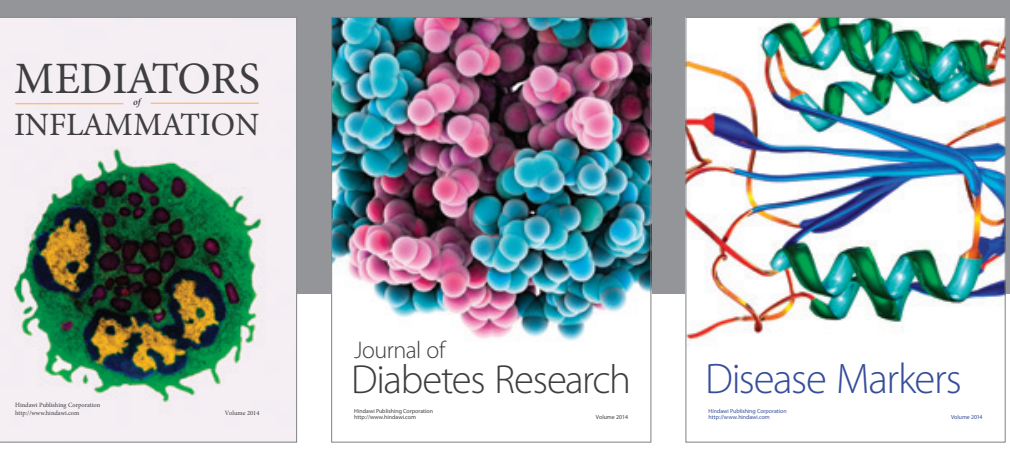

Disease Markers

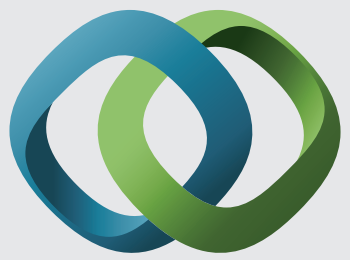

\section{Hindawi}

Submit your manuscripts at

https://www.hindawi.com
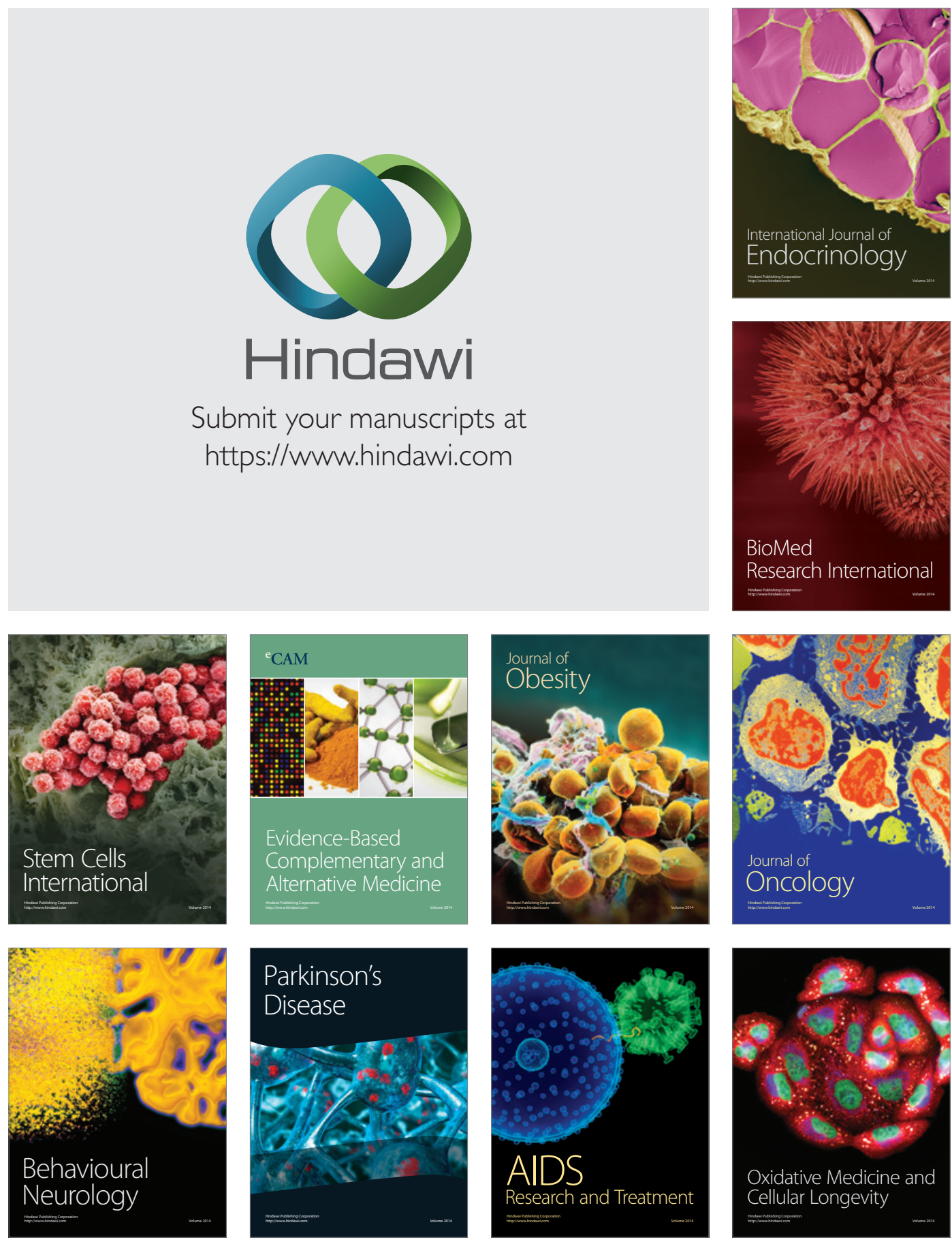